\title{
Parameters sensitivity of pharmacokinetics model parameters
}

\author{
V. Lifenko ${ }^{1}$, D. Voronov ${ }^{2}$, \\ ${ }^{1}$ Novosibirsk State University, Novosibirsk, Russia \\ ${ }^{2}$ Institute of Computational Mathematics and Mathematical Geophysics SB RAS, Novosibirsk, Russia \\ *e-mail:lera.lifenko@gmail.com
}

Key words: identifiability, pharmacokinetics, systems of ordinary differential equations, parameters sensitivity, inverse problem, singular value decomposition

Motivation and Aim: Systems of ordinary differential equations are an essential tool for modeling pharmacokinetic processes. In practice it is important not only to determine unknown parameters of the observed process but also to make sure that the solution is stable to errors in the experimental data [1].

Methods and Algorithms: Identifiability analysis is the first step in determining unknown parameters. Investigating parameters sensitivity to the data gives the necessary information for obtaining more stable solutions. A new technique for sensitivity analysis using singular value decomposition of the sensitivity matrix is shown. It was shown the influence of the stability of the parameters on the convergence of the gradient method. In addition, an overview of the methods for identifiability in nonlinear mixed effects models was given [2].

Results: A brief historical review of identifiability analysis methodologies for dynamic systems is presented. Methods of sensitivity analysis are reviewed in more details. Finally, some examples and numerical results for pharmacokinetic models of the kinetics and secretion of the C-peptide, the glucose-insulin control model, and the pharmacokinetics model of the Digoxin are presented.

Conclusion: Qualitative analysis of the sensitivity of mathematical models allows to avoid receiving unhelpful data and unnecessary expenses for experiments. Therefore, by investigating dynamical systems using the methods of identifiability analysis, we find sensitive parameters. Applying bounding conditions on parameters, or fixing them, we get a more stable problem.

Acknowledgements: This work was supported by RFBR grant No. 17-31-50060.

References:

1. Hongyu M. et al. (2011) On Identifiability of Nonlinear ODE Models and Applications in Viral Dynamics.

2. Lavielle M., Aarons L. (2016) What do we mean by identifiability in mixed effects models? 